\title{
A prática do enfermeiro na implementação de políticas públicas para a população negra: à luz da ética feminista'
}

\section{The practice of nurses in the implementation of public policies for the black population: in the light of feminist ethics}

\author{
Lilian Cristina Rezende ${ }^{a}$ \\ (iD) https://orcid.org/0000-0003-0869-0205 \\ E-mail: lilianc.enfळgmail.com \\ Carolina da Silva Caram ${ }^{a}$ \\ (D) https://orcid.org/0000-0001-6219-3301 \\ E-mail: caram.caroløgmail.com \\ Luana Silva Rezende ${ }^{a}$ \\ (iD) https://orcid.org/0000-0003-1551-4082 \\ E-mail: luanasilvarezendeœgmail.com \\ Thallison Carlos Campos Santos ${ }^{a}$ \\ (D) https://orcid.org/0000-0002-2390-3259 \\ E-mail: thallisonı \\ Beatriz Santana Caçador ${ }^{b}$ \\ (iD) https://orcid.org/0000-0003-4463-36II \\ E-mail: bya.cacador@gmail.com

\section{Maria José Menezes Brito ${ }^{a}$} \\ (D) https://orcid.org/0000-0001-9183-1982 \\ E-mail: mariajosejobrito®gmail.com \\ aniversidade Federal de Minas Gerais. Escola de Enfermagem. \\ Belo Horizonte, MG, Brasil. \\ bUniversidade Federal de Viçosa. Departamento de Medicina e \\ Enfermagem. Viçosa, MG, Brasil.
}

\section{Correspondência}

Lilian Cristina Rezende

Universidade Federal de Minas Gerais. Escola de Enfermagem. Departamento de Enfermagem Aplicada. Avenida Alfredo Balena, 190, 5 andar, sala 514. Belo Horizonte, MG, Brasil. CEP: 30130-100.

\section{Resumo}

Este estudo analisou as contribuições da prática do enfermeiro na implementação da Política Nacional de Saúde Integral da População Negra em comunidades quilombolas, sob o prisma da ética feminista. Estudo de caso único e integrado de abordagem qualitativa fundamentado na teoria da ética feminista. A coleta de dados ocorreu entre fevereiro e junho de 2018 por meio de entrevistas realizadas com sete enfermeiros da Estratégia Saúde da Família, que atuam em comunidades quilombolas na região metropolitana de Belo Horizonte, MG, Brasil. Os dados foram submetidos à análise de conteúdo com auxílio do software ATLAS. ti, versão 8. Os resultados apontaram contribuições da prática do enfermeiro na implementação da PNSIPN e estão apresentados pelas seguintes categorias: criação de vínculo; enfermeiro na comunidade e; reconhecimento das particularidades da comunidade. Tais aspectos se mostraram importantes para a implementação efetiva da Política Nacional de Saúde Integral da População Negra na comunidade.

Palavras-chave: Enfermagem; Ética Feminista; Estratégia Saúde da Família; Grupo com Ancestrais do Continente Africano; Grupos Minoritários, Políticas Públicas de não Discriminação.

\footnotetext{
1 Financiamento da pesquisa: Capes, CNPq, Fapemig, "Programa Pesquisa para o SUS: gestão compartilhada em saúde (PPSUS), apoio de SCTIE/MS, SES/MG e secretarias municipais da Região Metropolitana de Belo Horizonte (Belo Horizonte, Contagem, Pedro Leopoldo, Jaboticatubas e Brumadinho). CNPq: Processo n $409098 / 2018-3$, Chamada Universal 2018. Fapemig: Processo n APQ-03779-17-007/2017Programa de Pesquisa para o SUS-PPSUS. Capes.
} 
This qualitative integrated single-case study analyzed the role of nursing professionals in implementing the National Policy for the Integral Health of the Black Population in quilombola communities, based on the Feminist Ethics approach. Data collection took place between February and June 2018 by means of interviews conducted with nurses from the Family Health Strategy working in quilombola communities in the Metropolitan Region of Belo Horizonte, Minas Gerais, Brazil. The data underwent Content Analysis, via ATLAS.ti software, version 8. Results show that nursing professionals contributed to implementing the PNSIPN, acting on the following categories: establishing bonds; nurse in the community; recognizing the community's specificities. Such aspects are important to implement the National Policy for the Integral Health of the Black Population in the community. Keywords: Nursing; Feminist Ethics; Family Health Strategy, African Continental Ancestry Group; Minority Group; Public Nondiscrimination Policies.

\section{Introdução}

A prática do enfermeiro na Estratégia de Saúde da Família (ESF) se consolida de modo dialógico e interacional entre a equipe e os usuários, atrelada a uma prática reflexiva capaz de reconhecer o indivíduo, família e coletividades, e transformar a realidade social (Corrêa; Acioli; Tinoco, 2018). A concepção de prática, para fins desse estudo, será fundamentada na perspectiva epistemológica da ética feminista que tem como objeto as relações produzidas na interseccionalidade de grupos minoritários (Walker, 2007).

A prática, na perspectiva da ética feminista, acontece de forma colaborativa entre os sujeitos envolvidos, baseada na construção compartilhada e negociada de responsabilidades e reconhecimento das desigualdades e da diversidade cultural. Ela é alicerçada em comportamentos éticos advindos das experiências vividas entre os sujeitos, abrangendo saberes normativos, teóricos, políticos, empíricos, éticos e morais (Walker, 2007). Considerar, então, essa concepção de prática aplicada à do enfermeiro na ESF implica assumir que o cuidado é centrado nas relações entre os sujeitos e que, por isso, é capaz de transformar os seus modos de vida (Ferraccioli; Acioli, 2017).

Para que a prática do enfermeiro da ESF se conforme nesse constructo adotado é necessária a aproximação social e cultural do profissional com a população adscrita e o desenvolvimento da escuta atenta e do acolhimento ao usuário, de forma que ele se sinta confiante e seguro para expor suas dúvidas, medos e anseios (Corrêa; Acioli; Tinoco, 2018). Nessa relação, a prática baseada na colaboração e cooperação encontrará espaço para se concretizar.

Adentrando o universo das especificidades históricas e culturais do território brasileiro, nos deparamos com as iniquidades em saúde e a diversidade cultural, as quais, entre outros aspectos, se configuram como importantes desafios para a consolidação da prática do enfermeiro. Nessa perspectiva, destacam-se as particularidades das comunidades quilombolas, as quais são conformadas, em sua maioria, por grupos descendentes do continente africano que se organizam coletivamente em territórios delimitados (Brasil, 2017). 
A atuação do enfermeiro, a fim de superar as iniquidades étnico-raciais vivenciadas pelas comunidades quilombolas, precisa ser fundamentada na Política Nacional de Saúde Integral da População Negra (PNSIPN). A PNSIPN, instituída em 2009, propõe a elaboração e implementação de mecanismos e estratégias de não discriminação (Brasil, 2017). Contudo, estudo aponta que existem fragilidades na implementação da PNSIPN devido ao desconhecimento - por partes dos profissionais e da comunidade - sobre a sua existência (Franchi et al., 2018) e de práticas incoerentes com suas diretrizes (Brasil, 2017).

Para fins desse estudo parte-se do pressuposto que a prática do enfermeiro na ESF é capaz de minimizar essas fragilidades quando reconhece a diversidade cultural e histórica que compõe a sociedade.

Diante do exposto, a pergunta norteadora do estudo foi: como a prática do enfermeiro contribui na implementação de políticas públicas de saúde para a população negra? O objetivo do presente estudo foi analisar as contribuições da prática do enfermeiro na implementação da Política Nacional de Saúde Integral da População Negra em comunidades quilombolas, sob o prisma da ética feminista.

\section{Método}

Trata-se de um estudo de caso único e integrado de natureza qualitativa. Tal abordagem propicia a compreensão de aspectos subjetivos e complexos dos acontecimentos vividos no cotidiano com base em descobertas empíricas, com perspectiva interpretativa da visão de mundo e sua manifestação (Minayo, 2017; Yin, 2017).

Assume-se como caso do estudo a prática do enfermeiro da ESF no contexto de comunidades quilombolas. As comunidades quilombolas representaram cada subunidade integrada de análise, o que permite a triangulação das informações e o aprofundamento e compreensão de diferentes realidades sobre um mesmo objeto. A teoria da ética feminista, de Margaret Urban Walker, foi assumida como perspectiva analítica do caso em questão. Essa proposição teórica utilizada apresenta questões centrais capazes de analisar resultados empíricos complexos e contemporâneos, que são base para uma generalização analítica por permitir avanços teóricos (Yin, 2017). 0 estudo foi delineado conforme o checklist para os estudos qualitativos baseados nas diretrizes RATS (Clark, 2003).

O cenário do estudo foi constituído por sete ESF adscritas às comunidades quilombolas certificadas pela Fundação Cultural Palmares (FCP) (Brasil, 2018), alocadas na Região Metropolitana de Belo Horizonte (RMBH), Minas Gerais, Brasil. Os participantes desse estudo corresponderam a sete enfermeiros da equipe das ESF que atuavam nas comunidades quilombolas da RMBH. O critério de inclusão dos participantes foi atuar no mínimo seis meses na ESF.

A coleta de dados foi realizada no período de fevereiro a junho de 2018, mediante entrevista individual orientada por roteiro semiestruturado, conduzida por pesquisador externo e sem vínculo com o serviço. As questões abordavam sobre o conhecimento acerca da comunidade, das políticas públicas voltadas para o atendimento à população negra, das ações desenvolvidas na ESF e sobre as dificuldades e facilidades do cotidiano. Para a realização das entrevistas, os enfermeiros foram contatados pelo telefone e, após o aceite em participar do estudo, foi agendado dia, horário e local indicados por eles. As entrevistas foram gravadas e transcritas e seu tempo médio foi de 30 minutos. $\mathrm{Na}$ ocasião da entrevista, foram explicados os objetivos, riscos e benefícios da pesquisa e o Termo de Consentimento Livre Esclarecido (TCLE) foi lido e assinado por todos os participantes.

Os dados empíricos oriundos dos depoimentos dos enfermeiros foram submetidos à análise de conteúdo, seguindo os três polos cronológicos, a saber: pré-análise; exploração do material e tratamento dos resultados; inferência e interpretação (Bardin, 2011). Essa análise inicialmente foi realizada por subunidades e depois as informações foram trianguladas a fim de promover integração entre o caso em questão. Utilizou-se como suporte para a análise o Software Atlas.ti ${ }^{\circledR}$ versão 8 , por meio do qual os codes foram agrupados nas seguintes categorias temáticas: "criação de vínculo"; "enfermeiro na comunidade" e "reconhecimento das particularidades da comunidade". A ferramenta operacional Software Atlas.ti ${ }^{\circledR}$ proporcionou 
agilidade e segurança no tratamento e organização dos dados, propiciando a indexação, a busca e a teorização (Brito et al., 2017).

O estudo respeitou as exigências formais preconizadas pela Resolução n ${ }^{0}$. 466/12, do Conselho Nacional de Saúde, sendo aprovado pelo Comitê de Ética em Pesquisa da Universidade Federal de Minas Gerais e autorizado mediante Termo de Anuência pelas Secretarias Municipais de Saúde dos municípios. Foram preservados todos os direitos aos participantes, garantindolhes a liberdade de participar ou recusar, bem como de retirar seu consentimento no decorrer do trabalho por intermédio de manifestação expressa, livre e esclarecida. Para garantir o anonimato, as entrevistas receberam a denominação de "ENF", seguida do número aleatório de 1 a 7.

\section{Resultados}

Os resultados apontaram contribuições da prática do enfermeiro na implementação da PNSIPN e estão apresentados pelas seguintes categorias: criação de vínculo; enfermeiro na comunidade e reconhecimento das particularidades da comunidade. As categorias serão apresentadas de forma contínua e conectadas, uma vez que a prática do enfermeiro configura-se como transversal e relacional, baseado nos pressupostos da ética feminista. A respeito da criação de vínculo identificou-se a necessidade de fortalecer a relação com a comunidade a fim de encontrar meios para efetivar a PNSIPN. A categoria enfermeiro na comunidade perpassa pelas relações construídas e pela valorização da cultura por parte do profissional. E, por fim, a categoria "reconhecimento das particularidades da comunidade" demonstra a necessidade do enfermeiro se aproximar da comunidade para fortalecer o cuidado como potencial de transformação das condições de vida, da formação política e cidadã da população em prol dos seus direitos sociais.

O vínculo com a população é apontado por ENF6 como importante estratégia para a proposição de ações coerentes com os princípios da ESF e da PNSIPN, ampliando o acesso do usuário e contribuindo para que ele reflita sobre sua saúde e eventual adoecimento.
A importância maior é o vínculo que você cria com o paciente e esse vínculo você pode trabalhar prevenção, o paciente te escuta, por mais que na hora ele não te responda nada, mas você planta a sementinha. (ENF6)

Uma das formas de criação e de fortalecimento do vínculo é o enfermeiro na comunidade, em que ele reconhece e valoriza a cultura e os modos de viver da população adscrita à ESF. A esse respeito, ENF7 relata que a equipe de saúde promove eventos temáticos na unidade de saúde com abordagem das iniquidades sociais sofridas pela comunidade quilombola.

Temos implantado aqui o Dia da Consciência Negra, tem alguns anos que é comemorado. Então, a gente faz um evento aqui mesmo na unidade, com a participação do pessoal do quilombo, tem música, cada ano a gente propõe um tema diferente relacionado às questões sociais que observamose que eles nos trazem. (ENF7)

Esses eventos, promovidos pelos enfermeiros da ESF, são espaços de divulgação da cultura e de outras particularidades da população. A participação efetiva do enfermeiro e próxima da comunidade demonstra o compromisso e a responsabilidade do profissional com grupos minoritários e a construção compartilhada das ações entre eles, promovendo a discussão do racismo e da saúde da população negra, minimizando as iniquidades étnico-raciais, como preconizado pela PNSIPN.

Arelação entre enfermeiro e comunidade se destaca nas falas de $E F_{5}$ e ENF7, os quais apontam que a relação produzida no encontro entre eles promove $\mathrm{o}$ acolhimento e, assim, a resposta às necessidades.

As pessoas são muito carentes. Tem dia que as pessoas vêm aqui e não querem nada, só querem uma palavrae já saem daqui satisfeitos. A enfermagem também faz um pouco disso, um trabalho de psicologia também, de conversar, de ouvir, às vezes, o paciente só queria ser ouvido, está se sentindo sozinho, carente ( $\left.\mathrm{ENF}_{5}\right)$. É uma carência enorme, de certa forma, a gente ajuda um pouco as pessoas, não só na parte assistencial dos atendimentos que eu faço, mas um pouco de atenção que eu consigo dar, uma palavra. (ENF7) 
$\mathrm{O}$ (re)conhecimento da comunidade pelo enfermeiro gera entendimento da realidade experienciada e conduz o seu comportamento ético e moral diante das fragilidades dos grupos minoritários.

A prática da enfermagem foi destacada por ENF3 e ENF6, com ênfase em sua potencialidade transformadora da realidade da comunidade quilombola. Para os participantes, as mudanças de comportamento e dos modos de viver da população, no que concerne às suas questões de saúde, estão ligadas à relação de confiança estabelecida com o enfermeiro presente na comunidade.

A gente modifica muito a realidade da população. Você consegue traçar estratégias de atendimento e de execução. É muito diferenciado, o enfermeiro na Atenção Básica, na ESFe aqui nessa unidade émuito clara a credibilidade com relação ao enfermeiro, traçar a execução do serviço bem feito é a gente que traça. (ENF3) $O$ enfermeiro pode transformar o jeito do paciente olhar para a sua saúde, às vezes o que ele acha que é ruim, mas se ele começar a ver que tem um outro lado que vai trazer benefício, ele entende e começa a mudar estilo de vida e ver o que pode fazer para ser mais saudável. (ENF6)

A conformação da prática do enfermeiro centrado no indivíduo e na coletividade permite com que ele promova a transformação do estilo de vida dessas comunidades, pois é capaz de visualizar a necessidade do outro e buscar por respostas efetivas.

Cabe salientar que as necessidades da comunidade não se restringem as questões de saúde. Por isso, a prática do enfermeiro deve ser baseada no reconhecimento das particularidades da comunidade para o desenvolvimento da formação política e cidadã da população, como menciona ENF2. Ele aponta que o enfermeiro é considerado importante referência para a orientação de questões ligadas aos direitos da população e ao acesso aos mesmos, transcendendo o âmbito da saúde.

Eu acho que a comunidade precisa de um estímulo, um incentivo, tem gente que é muito tímido, muito quieto, muito calado, mas tem alguns que são mais envolvidos na comunidade. A gente sente que a gente não tem força, eles não sabem por onde começar.
Igual assim, 'eu tenho direito, direito a quê, então onde procuro esse direito?' Eles não têm isso, eles ficam muitas vezes querendo saber quem que eu tenho que procurar, o enfermeiro pode estimulá-los, orientá-los a entender seus direitos. Eles confiam muito em mim. (ENF2)

Os resultados apontaram a responsabilidade e a potencialidade da prática do enfermeiro como ação política transformadora da realidade social da comunidade quilombola com importantes contribuições na implementação da PNSIPN.

\section{Discussão}

A PNSIPN insere, no cenário das políticas públicas no Brasil, ações destinadas à promoção da saúde da população negra, focalizando suas especificidades e vulnerabilidades. Com seu caráter transversal no combate à discriminação étnica e racial nos serviços públicos de saúde do SUS, a PNSIPN busca sanar as necessidades de saúde da população negra e garantir a integralidade dos cuidados ao ampliar o acesso desse grupo populacional a serviços de saúde de qualidade (Brasil, 2017).

A implementação da PNSIPN é fundamental, não somente para o combate à discriminação nos serviços do SUS, mas também para assegurar a efetividade do alcance aos direitos da população negra, tendo em vista as suas lutas históricas em prol do respeito à sua cidadania e suas necessidades (Gomes et al., 2017). Na perspectiva da ética feminista, combater as iniquidades está intrinsecamente relacionado à crítica e mudança social que podem ser promovidas pelos sujeitos (Walker, 2007). A esse respeito percebeu-se nos resultados do presente estudo que o enfermeiro reconhece as necessidades da população e assume a corresponsabilização das atitudes que garantem os direitos da população negra.

Tendo em vista que a implementação da PNSIPN envolve o compromisso social do enfermeiro é primordial que ele estabeleça vínculo com a população para que ocorra, de fato, a efetivação das práticas de cuidado preconizadas pela PNSIPN, com ênfase para a promoção da saúde, a qual se destaca como eixo estruturante para seu vínculo com a comunidade. Nessa ótica, abre-se espaço para 
a construção da prática sob a égide da ética feminista, com destaque para a cooperação e negociação, em um processo intenso de (re conhecimentos para efetivar o compartilhamento de saberes e responsabilidades entre os atores sociais em um dado contexto (Walker, 2007). Os resultados demostraram que a relação estabelecida com a população é capaz de melhorar a adesão deles às propostas de cuidado, as quais são, cotidianamente e de forma participativa, construídas e reconstruídas.

A prática do enfermeiro precisa, pois, ser norteada pelo cuidado centrado no paciente/ família/comunidade e fundamentada em princípios da humanização como a escuta ativa, promotora do encontro com o profissional (Oliveira et al., 2018). No presente estudo, observou-se que esse encontro fortalece o vínculo, promovendo o acolhimento e desenvolvendo a responsabilidade moral do enfermeiro, em consonância com as reais necessidades da comunidade.

A responsabilidade moral do profissional se dá quando a pessoa é capaz de apreender o contexto social e moral de um grupo e de compreender seus valores e modos de viver, tecendo assim, responsabilidades por aquilo que apreende (Walker, 2007). Nesse sentido, a prática do enfermeiro demanda reflexões sobre a complexidade das questões sociais e da saúde, considerando a diversidade da sociedade nos tempos atuais (Schveitzer; Zoboli; Vieira, 2016). Ainda, o enfermeiro precisa resgatar a sua condição de sujeito-cidadão para que ele desenvolva seu potencial transformador da realidade da comunidade quilombola. Dessa forma, ele precisa compartilhar com a comunidade a sua prática, para que ela seja protagonista da própria saúde, sendo que a relação construída entre eles produz acolhimento.

$\mathrm{O}$ acolhimento, nesse sentido, foi percebido nos resultados do estudo como forma de ampliação do acesso e da valorização da população. Trata-se de um meio para proporcionar melhorias no relacionamento entre profissionais e usuários, transcendendo a mera forma de organização do trabalho, proporcionando autonomia dos sujeitos por meio do diálogo e da escuta qualificada (Schveitzer; Zoboli; Vieira, 2016). $\mathrm{Na}$ perspectiva da ética feminista a prática se conforma a partir da moralidade, o que significa reconhecer os sujeitos de grupos minoritários em seu contexto social, promovendo autonomia e responsabilidade moral por meio da experiência social deles (Walker, 2007). Esse contexto social é permeado por injustiças que destituem a sociedade da humanidade, tornando a realização de práticas pertinentes aos diversos grupos sociais um desafio para os profissionais (Walker, 2007).

As injustiças geram situações de vulnerabilidade vivenciadas por esse grupo populacional, como opressão, cerceamento dos seus direitos, bem como o racismo estrutural, tornando-se importante que o enfermeiro assuma em sua prática o acolhimento como dispositivo de escuta ativa, uma estratégia para acessar as necessidades de saúde, as quais extrapolam as demandas explicitadas pelos usuários (Melo; Cecílio; Andreazza, 2017; Ayres; Castellanos; Baptista, 2018). Na ética feminista, (re)conhecer as situações de vulnerabilidades e iniquidades de uma população específica, bem como sua diversidade cultural e particularidades do contexto social são aspectos fundamentais para a superação de práticas de exclusão (Walker, 2007).

Os resultados ressaltaram a importância do reconhecimento, por parte do enfermeiro, de que as carências e as dificuldades das comunidades quilombolas se configuram como potenciais dispositivos para o alcance de superações. Dessa forma é esperado que esse profissional seja capaz de realizar ações contínuas que proporcionem a sua própria transformação e a da realidade social (Corrêa; Acioli; Tinoco, 2018; Ferreira; Périco; Dias, 2018). Como exemplo de ações inovadoras e transformadoras da realidade, os participantes relataram a realização de eventos temáticos na unidade de saúde com foco na comunidade quilombola. A criação e realização desses eventos e espaços culturais é coerente com os objetivos da PNSIPN, haja vista a necessidade de comprometimento no combate às iniquidades de ordem socioeconômica, cultural e étnico-racial que atingem a população negra brasileira (Brasil, 2017). Destaca-se a importância de práticas que extrapolam ações pragmáticas definidas verticalmente e que consigam dar respostas ampliadas às reais demandas da população (Corrêa; Acioli; Tinoco, 2018).

Esse estudo contribui para a valorização da enfermagem no campo da ciência ao possibilitar a reflexão das contribuições da prática do enfermeiro 
em grupos minoritários, no que concerne ao (re)conhecimento de suas singularidades e ao compromisso social, especialmente com as comunidades quilombolas. Ainda, a contribuição se dá ao instigar os enfermeiros sobre a importância de romper as barreiras para promover a equidade, bem como atentar para um posicionamento sociopolítico que implique na implementação de políticas essenciais para a população, conferindo visibilidade social à profissão.

Infere-se que a prática dos enfermeiros se configura como meio de efetivar a implementação de políticas, como a PNSIPN, uma vez que ela traz elementos que superam o modelo biomédico, ampliando a noção de cuidado para as questões que envolvem as iniquidades vivenciadas por essa população. Tratase, pois, do posicionamento político assumido pelo enfermeiro, o qual implica na capacidade de articular os diferentes saberes e atribuições e emitir juízo próprio que responda às necessidades do cenário social, contribuindo para o fortalecimento da profissão (Melo; Cecílio; Andreazza, 2017).

As limitações do estudo pautam-se no limite geográfico e na limitação do uso do referencial da ética feminista. Sobre o limite geográfico a escolha foi a região metropolitana de Belo Horizonte. Sugere-se a realização de outros estudos em variadas regiões. Como recomendação, seria interessante a realização de um estudo etnográfico para melhor apreender as especificidades da comunidade e o desenvolvimento da prática do enfermeiro na ESF. A respeito da limitação teórica refere-se que a concepção de prática na teoria da ética feminista exige o cuidado por parte de pesquisadores na adoção desse constructo para análise dos dados, uma vez que se trata de uma prática específica, desenvolvida no contexto de grupos minoritários, que exige colaboração e responsabilidade mútua entre os sujeitos, não sendo uma mera realização de atividades.

\section{Considerações finais}

O presente estudo revelou que o enfermeiro, mediante a efetivação de uma prática colaborativa e de responsabilidade mútua, contribui na implementação da Política Nacional de Saúde
Integral da População Negra. Ressalta-se que o delineamento metodológico foi adequado e auxiliou na compreensão do objetivo.

O pressuposto teórico da ética feminista utilizado foi adequado e revelou a importância de que a prática em grupos minoritários seja realizada sob os preceitos da ética feminista. Tais preceitos referem que a prática é baseada na responsabilidade moral, compartilhada e negociada entre os sujeitos, os quais precisam reconhecer as iniquidades e a diversidade cultural presentes nos grupos minoritários.

Os resultados mostraram que a prática alicerçada na interrelação com a comunidade de forma a criar vínculos, reconhecer e valorizar a cultura e a história, possibilitar a formação política e de cidadania, bem como realizar o acolhimento para a que PNSIPN seja de fato implementada. Além disso, esses resultados trouxeram subsídios para auxiliar na formulação de novas políticas ao discutir aspectos da micropolítica que precisam ser considerados nessa construção.

A implementação e efetivação da PNSIPN se desenha nas nuances do cotidiano pelo envolvimento do enfermeiro na compressão do contexto sócio-histórico de desigualdades enraizadas da comunidade quilombola em busca da promoção e concretização de uma prática de saúde equânime.

\section{Referências}

AYRES, J. R.; CASTELLANOS, M. E. P; BAPTISTA, T. W. F. Interview with José Ricardo Ayres. Saúde e Sociedade, São Paulo, v. 27, n. 1, p. 51-6o, 2018. DOI: 10.1590/So104-12902018000002

BARDIN L. Análise de conteúdo. Lisboa:

Edições 70, 2011.

BRASIL. Ministério da Saúde. Política Nacional de Saúde Integral da População Negra: uma política do SUS. 3. ed. Brasília, DF: 2017. Disponível em: <https://bit.ly/2VooSVF>. Acesso em: 20 out. 2020. BRASIL. Ministério da Cultura. Fundação Cultural Palmares. Certidões expedidas às comunidades remanescentes de quilombos (CRQs) atualizada até a Portaria no. 88/2019, publicada no D. O.U. de 13/o5/2019. Brasília, DF, 2018. Disponível em: <https://bit.ly/2VbXqNB>. Acesso em: 20 out. 2020. 
BRITO, M. J. M. et al. Potentialities of Atlas.ti for data analysis in qualitative research in nursing. In: COSTA, A. P. et al. Computer supported qualitative research. Cham: Springer, 2017. p. 75-84. DOI: 10.1007/978-3-319-43271-7-7

CLARK, J. P. How to peer review a qualitative manuscript. In: JEFFERSON, T.; GODLEE, F. (Org.). Peer Review in Health Sciences. 2. ed. London: BJM Books, 2003, p. 219-235. Disponível em: <https://bit. ly/3k1wo5Z>. Acesso em: 11 maio 2021.

CORRÊA, V. A. F.; ACIOLI, S.; TINOCO, T. F. O cuidado do enfermeiro na Estratégia Saúde da Família: práticas e fundamentação teórica. Revista Brasileira de Enfermagem, Brasília, DF, v. 71, n. 6, p. 2767-2774, 2018. DOI: 10.1590/oo347167-2018-0383

FERRACCIOLI, P.; ACIOLI, S. As diferentes dimensões do cuidado na prática realizada por enfermeiros no âmbito da atenção básica. Revista de Pesquisa: Cuidado é fundamental online, Rio de Janeiro, v. 9, n. 1, p. 28-36, 2017. DOI: $10.9789 / 2175-5361.2017 \cdot v 911.28-36$

FERREIRA, S. R. S.; PÉRICO, L. A. D.; DIAS, V. R. F. G. The complexity of the work of nurses in Primary Health Care. Revista Brasileira de Enfermagem, Brasília, DF, v. 71, p. 704-709, 2018. DOI: 10.1590/0034-7167-2017-0471

FRANCHI, E. P. L. P. et al. A formação de profissionais de saúde no cuidado às comunidades quilombolas: um relato de experiência. Revista Brasileira de Medicina de Família e Comunidade, Rio de Janeiro, v. 13, n. 40, p. 1-11, 2018. DOI: $10.5712 / \mathrm{rbmfC}_{3}(40) 1620$
GOMES, I. C. R. et al. Implementação da política nacional de atenção integral à saúde da população negra na Bahia. Revista Baiana de Enfermagem, Salvador, v. 31, n. 2, 2017. Disponível em: <https://bit. ly/3hqX6U1>. Acesso em: 20 out. 2020.

MELO, S. M.; CECÍLIO, L. C. O; ANDREAZZA, R. Nem sempre sim, nem sempre não: os encontros entre trabalhadores e usuários em uma unidade de saúde. Saúde em Debate, Rio de Janeiro, v. 41, n. 112, p. 195-207, 2017. DOI: 10.1590/0103-1104201711216

MINAYO, M. C. S. Cientificidade, generalização e divulgação de estudos qualitativos. Ciência \& Saúde Coletiva, Rio de Janeiro, v. 22, n. 1, p. 16-17, 2017. DOI: 10.1590/1413-81232017221.30302016

OLIVEIRA, M. J. S. et al. A escuta ativa como estratégia de humanização da assistência em saúde. Saúde e Desenvolvimento Humano, Canoas, v. 6, n. 2, p. 33-38, 2018. DOI: http://dx.doi. org/10.18316/sdh.v6i2.4732

SCHVEITZER, M. C.; ZOBOLI, E. L. C. P.; VIEIRA, M. M. S. Nursing challenges for universal health coverage: a systematic review. Revista Latino-Americana de Enfermagem, Ribeirão Preto, v. 24, 2016. DOI: 10.1590/1518-8345.0933.2676

WALKER, M. U. Moral understandings: a feminist study in ethics. Oxford: Oxford University Press, 2007.

YIN, R. K. Case study research and applications: design and methods. Thousand Oaks: Sage publications, 2017.

\section{Contribuição dos autores}

Rezende, L. C. realizou a concepção e desenho do estudo, planejamento, coleta de dados, análise, interpretação, redação do trabalho e revisão final. Rezende, L. S. e Santos transcreveram, interpretaram e redigiram o trabalho. Caram e Caçador analisaram, interpretaram, redigiram o trabalho e fizeram a revisão final. Brito orientou a concepção e desenho do estudo e aprovou a versão final encaminhada.

Recebido: 17/05/2021

Reapresentado: 17/05/2021

Aprovado: 06/07/2021 